\title{
LATTICE DESIGN OF THE VLHC RINGS*
}

\author{
J. Johnstone, M. Syphers, FNAL, Batavia, IL 60510, USA \\ S. Peggs, S. Tepikian, BNL, Upton, NY 11973, USA
}

\begin{abstract}
The Very Large Hadron Collider (VLHC) is comprised of 2 rings, each having a $233 \mathrm{~km}$ circumference. The high-field (HF) ring is characterized by 90 o FODO cells constructed from separated-function magnets \& vertical beam separation. The low-field (LF) accelerator is also constructed from 90o cell, but these employ combined function "transmission line" magnets and horizontally separated beams. The optics of the 2 rings are virtually identical. The HF machine operates in the synchrotron radiation dominated regime. Doublet final focusing is employed to take advantage of the flat beam optics.
\end{abstract}

\section{MACHINE LAYOUTS}

It is envisioned that VLHC construction will be implemented in two phases, resulting in 2 rings within the same tunnel. The first phase will be a low-field ring, supporting $20 \times 20 \mathrm{TeV}$ collisions with a peak, initial luminosity of $10^{34} \mathrm{~cm}^{-2} \mathrm{sec}^{-1}$. The LF ring will be constructed almost entirely from very long, combinedfunction magnets. These magnets will have horizontallyseparated beams in two-in-one magnets with central fields of $2 \mathrm{~T}$. The second stage, high-field machine, will operate at up to $100 \times 100 \mathrm{TeV}$ with a peak luminosity of $2 \times 10^{34}$ $\mathrm{cm}^{-2} \mathrm{sec}^{-1}$. It will exclusively use strong, separated function magnets with vertically separated beams. The size of the arcs is determined primarily by the low bend field of the LF collider, while the lengths of the major straight sections are determined by the high magnetic rigidity of beams in the second stage collider.

Both low \& high field colliders are constructed from a few types of optical modules linked end to end. The length of each module is an integer multipole of $\mathrm{L}_{\mathrm{hc}}$ - the arc half-cell length. One advantage of modular construction is that the low \& high field lattices have almost identical footprints and fit in the same tunnel if corresponding modules are placed on top of each other. In the design studied here, the maximum radial offset between the machines is only $\pm 2.5 \mathrm{~mm}$ throughout the arcs. Another advantage is the ability to modify independently one particular module without adjusting any other modules. This is only possible if the arc half-cell length is an integer times the bunch spacing, because only then is the total circumference guaranteed to correspond to an integer number of evenly spaced bunches.

The minimum bunch spacing is taken to be one 53 MHz Tevatron RF wavelength:

$$
S_{B}=\frac{2000 \pi}{1113}=5.6453 \mathrm{~m}
$$

The half cell length is chosen to be $\mathrm{L}_{\mathrm{hc}}=24 * \mathrm{~S}_{\mathrm{B}}=$ $135.4865 \mathrm{~m}$. The maximum value of $\mathrm{L}_{\mathrm{hc}}$ is limited mainly by the need to protect the LF ring from vulnerability to systematic field harmonic errors.

\footnotetext{
* Work supported by the Universities Research Association, Inc., under contract DE-AC02-76CH00300 with the U.S. Dept. of Energy.
}

Dispersion suppresser modules (DS) are adopted following the SSC design, which was also used in the Fermilab Main Injector \& Recycler rings. With 90o of phase advance per arc cell, a dispersion suppressing module can be constructed from two cells, each of which is 3/4 the standard arc cell length and has 2/3 the bend.

Figure 1 illustrates how each collider is made of two major arcs connecting 2 clusters of straight sections. Only one cluster region - located at the Fermilab site - will be equipped with full interaction region (IR) \& utility region (UT) optics. The opposite cluster will have the same straight section lengths but, apart from a few specialized insertions, will be made from simple 90o FODO cells.

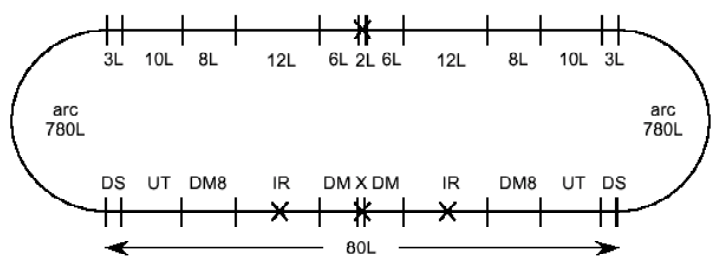

Figure 1: Schematic layout of the colliders. Lengths are given as multiples of the fundamental half cell length $L$.

Table 1. Fundamental lattice parameters common to both low $\&$ high field rings.

\begin{tabular}{|lrl|}
\hline Circumference & 233.037 & $\mathrm{~km}$ \\
Average arc Radius & 35.0 & $\mathrm{~km}$ \\
Number of IP's & 2 & \\
Half cell length & 135.4865 & $\mathrm{~m}$ \\
Half cell bend angle & 3.875 & $\mathrm{mrad}$ \\
Half cell count & 1720 & \\
Half cell harmonic & 24 & \\
Bunch spacing & $5.645 \mathrm{~m}$ \\
Number of buckets & 41280 & \\
\hline
\end{tabular}

Some of the fundamental parameters of the footprint are provided in Table 1. In the transition from Stage-1 to Stage- 2 operation the experiments will remain centered on the same interaction points (IP). It is therefore necessary to allow for a bypass to keep the low-field beams well clear of the experiments. This must also be done without changing the total low-field ring circumference. A sketch of the future by-pass appears in Figure 2.

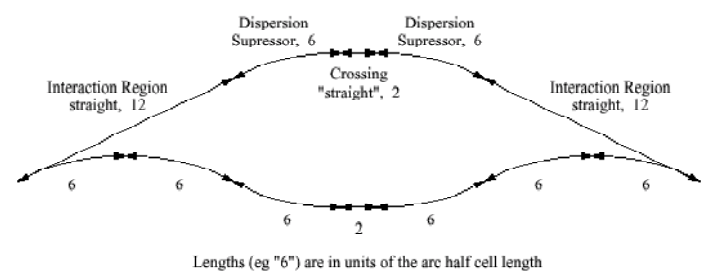

Figure 2: Future bypass of the LF ring around the HF experimental regions 


\section{LOW-FIELD LATTICE}

The bulk of the lattice is characterized by its construction from just three optical building blocks - arc, dispersion suppressing, and straight-section cells. The arc $\&$ suppresser cells consist of combined function gradient magnets, with space allocated at maximum- $\beta$ sites for 4 correction elements. Additional corrector space is available at the quarter-cell locations $(\beta \mathrm{x}=\beta \mathrm{y})$ for lumped correction of systematic multipoles [1].

\subsection{Interaction Regions}

The final foci in the IR's are anti-symmetric triplets, formed from single-bore, $300 \mathrm{~T} / \mathrm{m}$ magnets. Four additional quadrupole circuits, comprising double-bore, 70 $\mathrm{T} / \mathrm{m}$ magnets, are also used in optical matching. With a total of 6 independently-tunable quadrupole circuits available it is possible to match the four $\beta$ 's \& $\alpha$ 's from the IP to regular FODO cells, plus hold the phase advance $\Delta \mu$ constant across the IR through the squeeze from $\beta^{*}=$ $6.00 \mathrm{~m} \rightarrow 0.30 \mathrm{~m}$. Fixing $\Delta \mu$ eliminates the necessity of a special phase trombone to maintain the nominal operating point. Figure 3 shows the lattice functions through the IR during collisions.

The circulating beams are separated horizontally by 15 $\mathrm{cm}$ throughout the arcs \& $70 \mathrm{~T} / \mathrm{m}$ straight-section quads. Four dipoles, $13.6 \mathrm{~m}$ each @ $1.97 \mathrm{~T}$, situated between the Q3 \& Q4 quadrupoles bring the beams together at the entrance to the triplet for collisions at the IP. Dipoles downstream of the IP separate the beams again \& channel them back into the inner \& outer rings. A half-crossing angle of $77 \mu \mathrm{r}$ at the IP gives $10 \sigma$ separation between the beams at the first parasitic crossing.

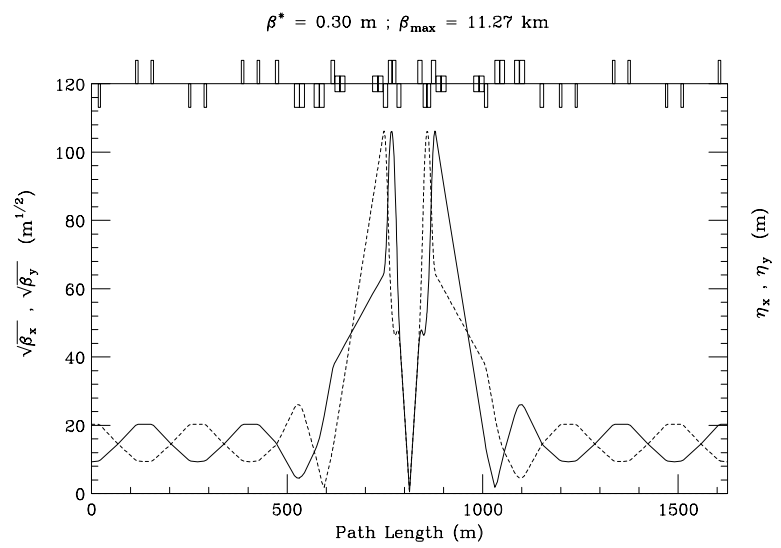

Figure 3: IR lattice functions at collision $\beta^{*}=30 \mathrm{~cm}$.

\subsection{Injection \& Abort Straight Sections}

To accommodate $900 \mathrm{GeV}$ injection from the Tevatron plus abort of $20 \mathrm{TeV}$ protons, 5 straight-section cells are modified as shown in Figure 4. A long drift space ( $377 \mathrm{~m})$ is created by triplet focusing to produce sufficient room for abort lambertsons (not shown) \& beam extraction.

Dipoles separate the circulating beams through the utility straight to make room for RF cavities downstream of the 2 nd triplet. Three $21.54 \mathrm{~m}$ dipoles at $2 \mathrm{~T}$ bend the beams apart by $40 \mathrm{~cm}$, followed immediately by 3 more dipoles that flatten out the trajectories. This pattern is reversed downstream of the RF to bring the beams back into standard double-bore magnets

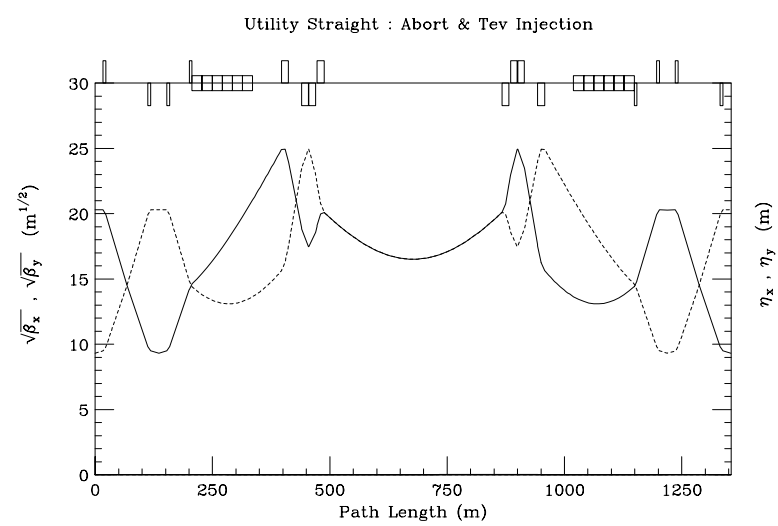

Figure 4: Layout of a utility straight supporting beam transfer from the Tevatron and $20 \mathrm{TeV}$ abort.

\subsection{Beam Crossovers}

With the beams separated horizontally in the arcs it is necessary to move the beams between the inner \& outer rings to maintain identical path lengths. In the LF ring 2 cross-over cells are inserted on opposite sides of the ring. Four rolled dipoles move the beams the $15 \mathrm{~cm}$ from one channel to the other. Each dipole is $36 \mathrm{~m}$ at $1.944 \mathrm{~T}$. The first dipole is rolled by $0.12 \mathrm{rad}$ to start separating the beams vertically, while bringing them closer together horizontally. The $2 \mathrm{nd}$ bend is rolled by $-0.12 \mathrm{rad}$ to flatten the vertical trajectory. At the crossing point the beams are separated vertically by $9 \mathrm{~mm}$. With $\beta y=165 \mathrm{~m}$, and $\varepsilon_{\mathrm{N}}=$ $1.5 \pi \mu \mathrm{m}$, this is $17.75 \sigma$ at the injection energy of 900 $\mathrm{GeV}$. Rolled dipoles after the crossing point remove the vertical offsets \& complete the $15 \mathrm{~cm}$ transverse transfer of beams to the opposite channel. One cross-over cell is shown in Figure 5.

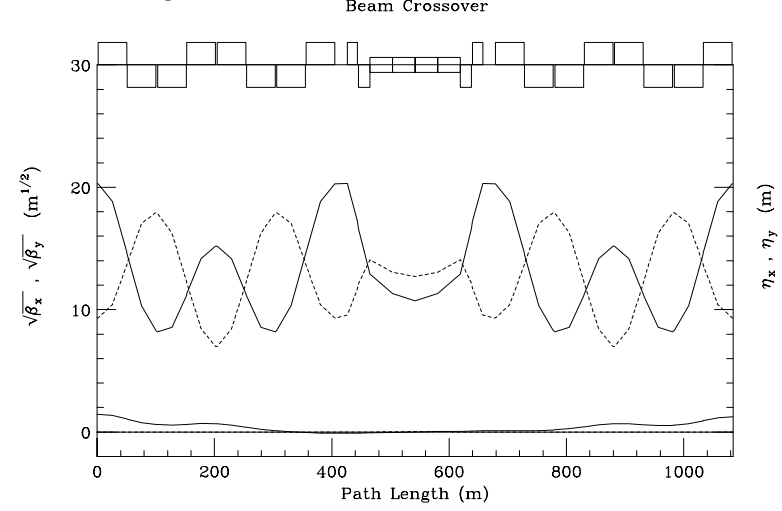

Figure 5. Cross-over cell with doublet focusing.

\section{HIGH-FIELD LATTICE}

The high-field VLHC ring will be the first cryogenic collider to operate in the synchrotron radiation dominated regime. With the radiation damping time being shorter than the storage time, the beam emittances evolve into an asymmetric state characterized by $\kappa \equiv \varepsilon_{\mathrm{y}} / \varepsilon_{\mathrm{x}}$. The HF ring should be able to achieve $\kappa<<0.1$ during a store, consistent with the results from conventional electron storage rings. 
It is the head on beam-beam interaction that sets the minimum horizontal emittance. Based on experience at the Tevatron \& SPS, the beam-beam limit is expected to be approximately $\xi \approx 0.008$. The horizontal \& vertical tune shift parameters in general are:

$$
\xi_{q}=\frac{r}{2 \pi} \cdot N \cdot \frac{\beta_{q}^{*}}{\sigma_{q} \cdot\left(\sigma_{x}+\sigma_{y}\right)}
$$

The beam-beam parameters are made equal for flat beams by making the ratio of $\beta^{*}$ 's also equal to $\kappa$ :

From which it follows:

$$
\kappa=\frac{\varepsilon_{y}}{\varepsilon_{x}}=\frac{\beta_{y}^{*}}{\beta_{x}^{*}}=\frac{\sigma_{y}^{*}}{\sigma_{x}^{*}}
$$

$$
\xi_{x}=\xi_{y}=\frac{1}{(1+\kappa)} \cdot \frac{r}{2 \pi} \cdot \frac{N}{\varepsilon_{x}}
$$

Figure 6 shows the horizontal $\&$ vertical emittances decreasing to plateau values of $\varepsilon_{\mathrm{X}} \& \varepsilon_{\mathrm{y}}$ consistent with the beam-beam limit.

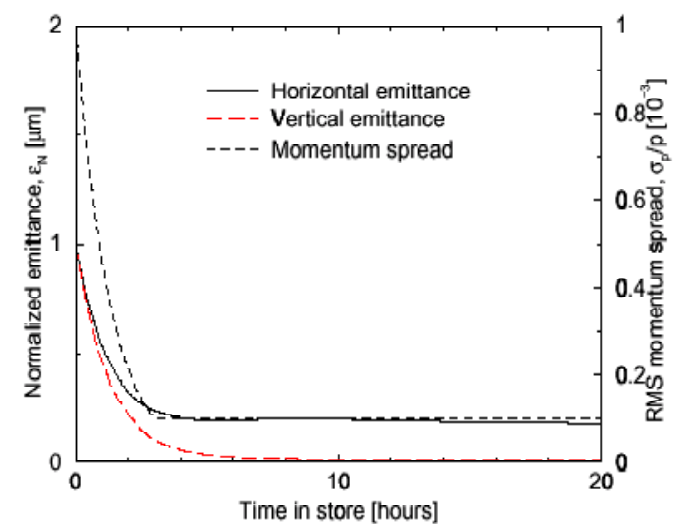

Figure 6: Evolution of the transverse emittances and the rms momentum spread during a store.

The instantaneous luminosity is:

$$
L=\frac{M}{4 \pi T} \cdot \frac{N^{2} \gamma}{\sqrt{\varepsilon_{x} \varepsilon_{y} \beta_{x}^{*} \beta_{y}^{*}}} \rightarrow \frac{M}{4 \pi T} \cdot \frac{N^{2} \gamma}{\kappa \varepsilon_{x} \beta_{x}^{*}}
$$

It is clear that with flat beams the value of $\beta^{*} x$ can be increased by a factor of about $1 / \kappa-$ significantly simplifying the optics - and reducing $\beta$ max by an order of magnitude. A selection of operational parameters with flat-beam optics is given in Table 2 .

Table 2. Flat beam parameters after 6 hours into the store, when both $\mathrm{x} \& \mathrm{y}$ beam-beam parameters are saturated.

\begin{tabular}{|lrl|}
\hline Flatness parameter, $\kappa$ & 0.1 & \\
Beam-beam parameter $\xi \mathrm{x}, \mathrm{y}$ & 0.008 & \\
Horizontal collision $\beta^{*}$ & $3.7 \mathrm{~m}$ \\
Vertical collision $\beta^{*}$ & $0.37 \mathrm{~m}$ \\
Horizontal maximum $\beta$ & $7.84 \mathrm{~km}$ \\
Vertical maximum $\beta$ & $10.75 \mathrm{~km}$ \\
Horizontal emittance $\varepsilon$ & $0.161 \mathrm{\mu m}$ \\
Vertical emittance $\varepsilon$ & $0.016 \mu \mathrm{m}$ \\
Collision beam size $\sigma$ & $2.53 \mathrm{\mu m}$ \\
Collision beam size $\sigma$ & $0.25 \mathrm{\mu m}$ \\
\hline
\end{tabular}

\subsection{Interaction Regions}

Doublet optics are more naturally suited to the flat beams of the high-field ring. Flat beams require the first quadrupole on both sides of the IP to be vertically focusing to both circulating beams. Thus, the optics must be symmetric across the IP, and the first quadrupole must be a 2-in-1 magnet.

The IR gives $30 \mathrm{~m}$ free space from the IP to the first magnetic element. The first magnet encountered is a highfield, small bore magnet of $16 \mathrm{~T}$ field. This can handle the beams until the total separation becomes $8 \mathrm{~mm}$. Then the beams enter a lower field, $12 \mathrm{~T}$, magnet with a larger bore. These beam separation dipoles bend the beam vertically. This crossing region requires that the beam separation at the first quadrupole be $30 \mathrm{~mm}$. With these dimensions the first quadrupole is limited to $400 \mathrm{~T} / \mathrm{m}$ gradient. As the beams separate, a higher quadrupole field can be attained with a limit of $600 \mathrm{~T} / \mathrm{m}$.

With this design, two interactions regions can fit in the on-site cluster region. The $\beta^{*}$ can be varied from 0.37 to $7.12 \mathrm{~m}$ vertically ( $\beta^{*}$ horizontally is 10 times larger). The maximum $\beta y$ is $10.6 \mathrm{~km}$ with the collision optics and only $760 \mathrm{~m}$ at injection. Figure 7 shows the collision optics of the HF interaction region.

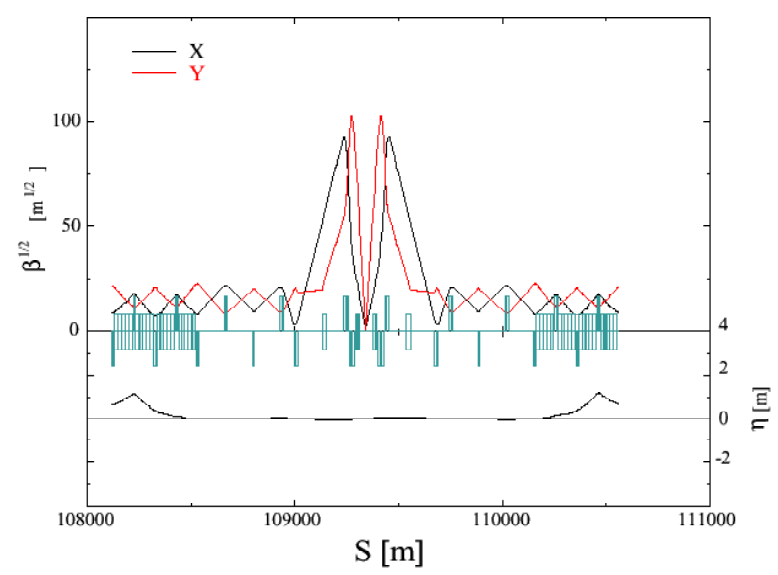

Figure 7: Lattice functions near the IP at collision.

\section{SUMMARY}

A concept for implementing the VLHC as a two-phase project is being developed. The $20 \mathrm{TeV}$ first phase machine would significantly extend the energy frontier beyond the LHC. IR optics are characterized by round beams \& triplet focusing. The low, 2 T, bend field of the arc magnets dictates the circumference of the VLHC tunnel. The high energy, $100 \mathrm{TeV}$, second phase machine would occupy this same tunnel. Synchrotron radiation damping in the HF ring determines the optimal optics configuration -- flat beams and doublet IR final focusing.

\section{REFERENCES}

[1] David Neuffer, "Lumped Correction of Systematic Multipoles in Large Synchrotrons", Particle Accelerators 23, 1988. 